\title{
A New Two-Parameter Burr-Hatke Distribution: Properties and Bayesian and Non-Bayesian Inference with Applications
}

\author{
Ahmed Z. Afify $\mathbb{D}^{1},{ }^{1}$ Hassan M. Aljohani, ${ }^{2}$ Abdulaziz S. Alghamdi $\mathbb{D}^{3},{ }^{3}$ Ahmed M. Gemeay, ${ }^{4}$ \\ and Abdullah M. Sarg ${ }^{1}$ \\ ${ }^{1}$ Department of Statistics, Mathematics and Insurance, Benha University, Benha 13511, Egypt \\ ${ }^{2}$ Department of Mathematics \& Statistics, College of Science, Taif University, P.O. Box 11099, Taif 21944, Saudi Arabia \\ ${ }^{3}$ Department of Mathematics, College of Science \& Arts, King Abdulaziz University, P.O. Box 344, Rabigh 21911, Saudi Arabia \\ ${ }^{4}$ Department of Mathematics, Faculty of Science, Tanta University, Tanta 31527, Egypt
}

Correspondence should be addressed to Ahmed Z. Afify; ahmed.afify@fcom.bu.edu.eg

Received 24 August 2021; Accepted 9 November 2021; Published 28 November 2021

Academic Editor: Barbara Martinucci

Copyright (C) 2021 Ahmed Z. Afify et al. This is an open access article distributed under the Creative Commons Attribution License, which permits unrestricted use, distribution, and reproduction in any medium, provided the original work is properly cited.

\begin{abstract}
This article introduces a two-parameter flexible extension of the Burr-Hatke distribution using the inverse-power transformation. The failure rate of the new distribution can be an increasing shape, a decreasing shape, or an upside-down bathtub shape. Some of its mathematical properties are calculated. Ten estimation methods, including classical and Bayesian techniques, are discussed to estimate the model parameters. The Bayes estimators for the unknown parameters, based on the squared error, general entropy, and linear exponential loss functions, are provided. The ranking and behavior of these methods are assessed by simulation results with their partial and overall ranks. Finally, the flexibility of the proposed distribution is illustrated empirically using two real-life datasets. The analyzed data shows that the introduced distribution provides a superior fit than some important competing distributions such as the Weibull, Fréchet, gamma, exponential, inverse log-logistic, inverse weighted Lindley, inverse Pareto, inverse Nakagami-M, and Burr-Hatke distributions.
\end{abstract}

\section{Introduction}

Survival and reliability analysis is an important area of statistics and it has various applications in several applied sciences such as engineering, economics, demography, medicine, actuarial science, and life testing. Different lifetime distributions have been introduced in the statistical literature to provide greater flexibility in modeling data in these applied sciences.

One of the important features of generalized distributions is their capability for providing superior fit for various life-time data encountered in the applied fields. Hence, the statisticians have been interested in constructing new families of distributions to model such data. Some recent notable families are the following: the exponential T-X [1], transmuted Burr-X [2], Marshall-Olkin Burr-III [3], Marshall-Olkin Burr [4], and log-logistic tan [5] families.
On the other hand, there are some useful techniques to add an additional parameter to extend and enhance the flexibility of the classical distributions such as the inverse-power (IP) transformation. Let $X$ and $Y$ be two random variables. The inverse transformation, say $X=Y^{-1}$, or the IP transformation, say $X=Y^{-(1 / \eta)}$, has been adopted by many authors to construct generalized inverted distributions. For example, the generalized inverse gamma [6], the inverse Lindley with two parameters [7], the inverse Lindley [8], the inverse-power Maxwell [9], the inverse-power Lindley [10], and inverse-power Lomax [11].

In this paper, we are motivated to propose a more flexible version of the Burr-Hatke $(\mathrm{BH})$ distribution to increase its flexibility in modeling real-life data. The $\mathrm{BH}$ model provides only a decreasing hazard rate (HR) shape; hence, its use will be limited to modeling the data that exhibits only 
increasing failure rate. The proposed distribution is called the inverse-power Burr-Hatke (IPBH) distribution. The IPBH model can accommodate right-skewed shape, symmetrical shape, reversed J shape, and left-skewed shape densities. Its $\mathrm{HR}$ can be an increasing shape, a unimodal shape, or a decreasing shape. The IPBH provides more accuracy and flexibility in fitting engineering and medicine data. The IPBH distribution was constructed using the inverse-power (IP) transformation.

Isaic-Maniu and Voda [12] proposed the $\mathrm{BH}$ distribution with shape parameter $\alpha$. Its cumulative distribution function $(\mathrm{CDF})$ has the following form:

$$
F(x ; \alpha)=1-\frac{\exp (-\alpha x)}{x+1}, \quad \alpha>0, x>0 .
$$

Its probability density function (PDF) takes the following form:

$$
f(x ; \alpha)=\frac{\exp (-\alpha x)(\alpha+\alpha x+1)}{(x+1)^{2}}, \quad \alpha>0, x>0 .
$$

We also considered ten various classical and Bayesian methods for estimating the IPBH parameters and provided detailed numerical simulations to explore their performances based on the mean square errors (MSE), mean relative estimates (MRE), and absolute biases (BIAS). The classical estimators proposed included the maximum product of spacing estimators (MPSE), Anderson-Darling estimators (ADE), Cramér-von Mises estimators (CVME), least-squares estimators (LSE), maximum likelihood estimators (MLE), right-tail Anderson-Darling estimators (RTADE), and weighted least-squares estimators (WLSEs). The Bayesian estimators of the IPBH parameters have been obtained under symmetric and asymmetric loss functions, namely, the square errors (SE), general entropy (GE), and linear exponential (LN) loss functions. We have compared the estimation methods by conducting extensive simulations study to explore their performances and to determine the best method of estimation, based on partial and overall ranks, which gives accurate estimates for the IPBH parameters.

It is shown empirically that the IPBH distribution can provide a more adequate fit than ten competing distributions, namely, the $\mathrm{BH}$ [12], Weibull (W), Fréchet (F), gamma $(G)$, exponential (E), inverse log-logistic (ILL) [13], inverse weighted Lindley (IWL) [14], inverse Lindley (IL) [14], inverse Pareto (IP) [15], and inverse Nakagami-M (INM) [16] distributions.

This article is outlined in the following eight sections. The IPBH distribution is defined in Section 2. Some of its properties are discussed in Section 3. In Section 4, seven classical approaches of estimation are explored. The Bayesian estimators of the IPBH parameters under three loss functions are discussed in Section 5. In Section 6, the performances of classical and Bayesian approaches of estimation are explored via simulations. The applicability and flexibility of the IPBH distribution are illustrated in Section 7 using two real-life datasets. Some useful conclusions are presented in Section 8.

\section{The IPBH Distribution}

By applying the IP transformation to the $\mathrm{BH}$ CDF (1), the $\mathrm{CDF}$ of the IPBH distribution follows (for $x>0$ ) as

$$
F(x ; \alpha, \eta)=\frac{\exp \left(-\alpha x^{-\eta}\right)}{x^{-\eta}+1}, \quad \alpha, \eta>0 .
$$

The corresponding PDF of the IPBH distribution reduces to

$$
f(x ; \alpha, \eta)=\frac{\eta \exp \left(-\alpha x^{-\eta}\right)\left[\alpha+(\alpha+1) x^{\eta}\right]}{x\left(x^{\eta}+1\right)^{2}}, \quad \alpha, \eta>0,
$$

where $\eta$ and $\alpha$ are shape parameters. The inverse BH (IBH) distribution follows simply as a special case by replacing $\eta=$ 1 in equation (4).

The survival function (SF) and HR function of the IPBH distribution take the following forms, respectively:

$$
\begin{aligned}
& S(x ; \alpha, \eta)=1-\frac{x^{\eta} \exp \left(-\alpha x^{-\eta}\right)}{x^{\eta}+1}, \\
& h(x ; \alpha, \eta)=\frac{\eta\left[\alpha+(\alpha+1) x^{\eta}\right]}{x\left(x^{\eta}+1\right)\left[\left(x^{\eta}+1\right) \exp \left(-\alpha x^{-\eta}\right)-x^{\eta}\right]} .
\end{aligned}
$$

Possible shapes of the density and HR functions of the IPBH distribution are displayed in Figures 1 and 2, respectively.

\section{Mathematical Properties}

In this section, some distributional properties are addressed.

3.1. Quantile Function. The quantile function (QF) of the IPBH distribution is derived from the CDF (3) as

$$
Q(p)=\left[\frac{1}{\alpha} W\left(\frac{\alpha \exp (\alpha)}{p}\right)-1\right]^{-1 / \eta}, \quad 0<p<1,
$$

where $W[\cdot]$ is Lambert function.

The three quartiles of the IPBH distribution follow directly from (6) with $p=0.25,0.5$, and 0.75 .

Assuming that $p$ uniform $(0,1)$, the QF (6) can be applied to generate random datasets of size $n$ from the IPBH distribution by the following formula:

$$
x_{i}=\left[\frac{1}{\alpha} W\left(\frac{\alpha \exp (\alpha)}{p}\right)-1\right]^{-1 / \eta}, \quad i=1,2, \ldots, n .
$$

Moreover, the QF (6) is used to determine the Bowley skewness, say SK, and Moors kurtosis, say KU, measured by the two following equations:

$$
\begin{aligned}
& \mathrm{SK}=\frac{Q(1 / 4)+Q(3 / 4)-2 Q(1 / 2)}{Q(3 / 4)-Q(1 / 4)}, \\
& \mathrm{KU}=\frac{Q(7 / 8)-Q(5 / 8)+Q(3 / 8)-Q(1 / 8)}{Q(6 / 8)-Q(2 / 8)} .
\end{aligned}
$$



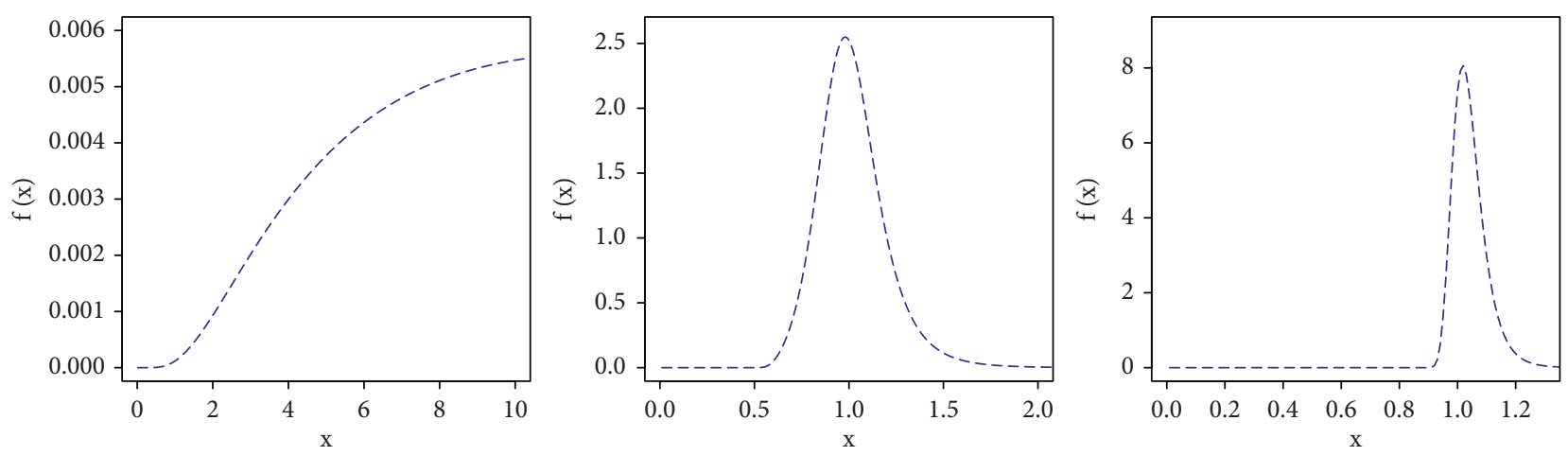

$--\alpha=10 \eta=0.5$

$---\alpha=0.01 \eta=10$

$--\alpha=0.75 \eta=25$
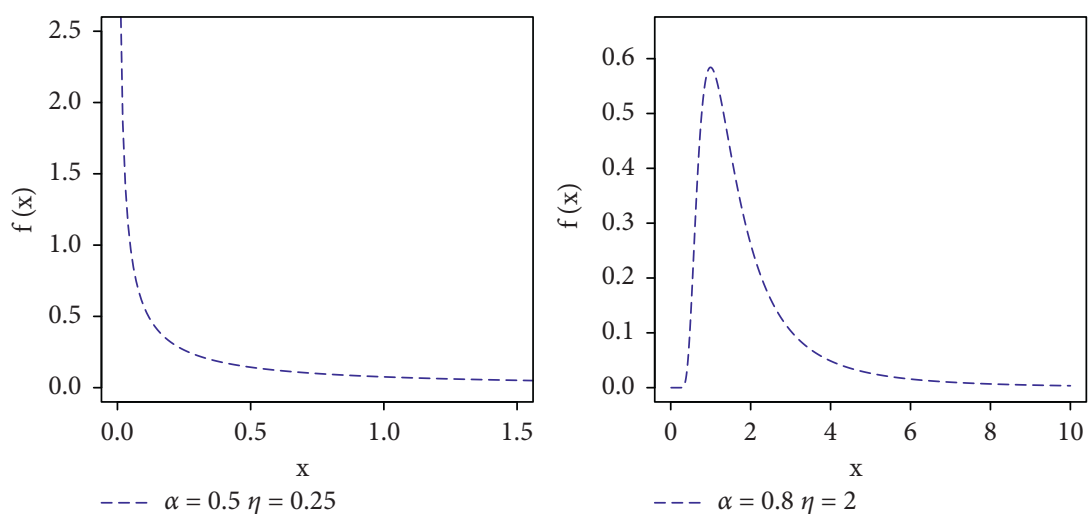

FIgUre 1: Possible density shapes of the IPBH distribution for several values of $\alpha$ and $\eta$.
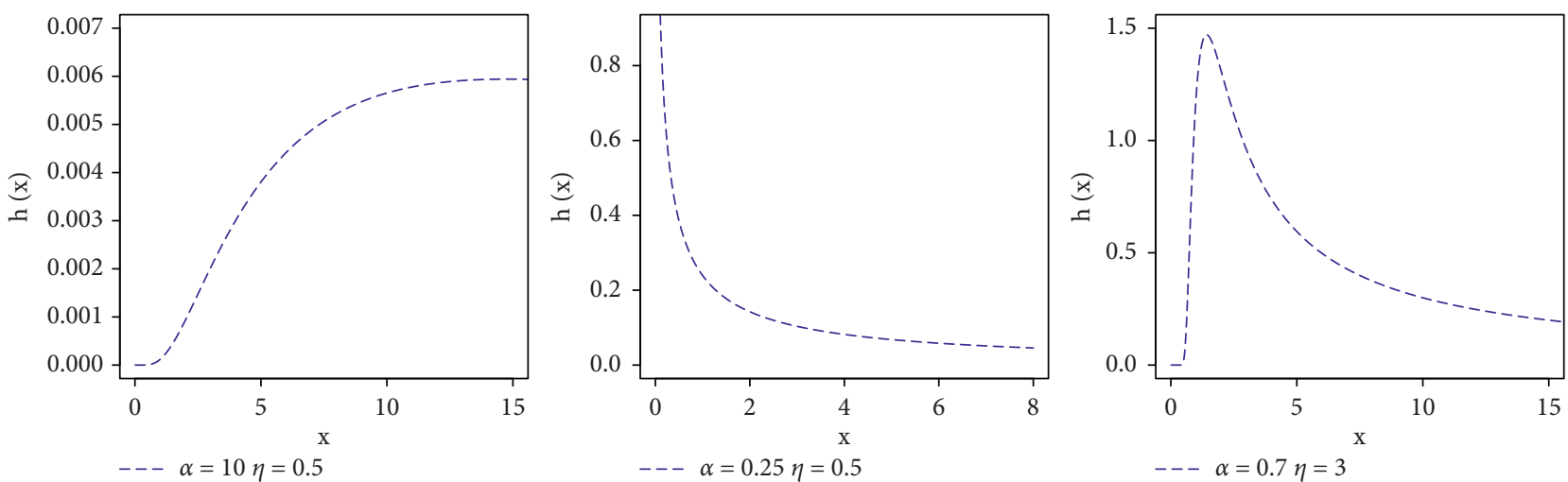

FIgUre 2: Possible failure rate shapes of the IPBH distribution for several values of $\alpha$ and $\eta$.

The shapes of SK and KU of the IPBH model for several values of $\alpha$ and $\eta$ are displayed in Figure 3.
3.2. Moments. The $r$ th moments of the IPBH distribution have the following forms:

$$
\begin{aligned}
\mu_{r}^{\prime} & =E\left(X^{r}\right)=\int_{0}^{\infty} x^{r} f(x) \mathrm{d} x=\int_{0}^{\infty} \frac{\eta x^{r-1} e^{-\alpha x^{-\eta}}\left[\alpha+(\alpha+1) x^{\eta}\right]}{\left(x^{\eta}+1\right)^{2}} \mathrm{~d} x \\
& =\sum_{k=0}^{\infty} \frac{(-1)^{k} \eta \alpha^{k}}{k !} \int_{0}^{\infty} \frac{x^{r-\eta k-1}\left[\alpha+(\alpha+1) x^{\eta}\right]}{\left(x^{\eta}+1\right)^{2}} \mathrm{~d} x
\end{aligned}
$$



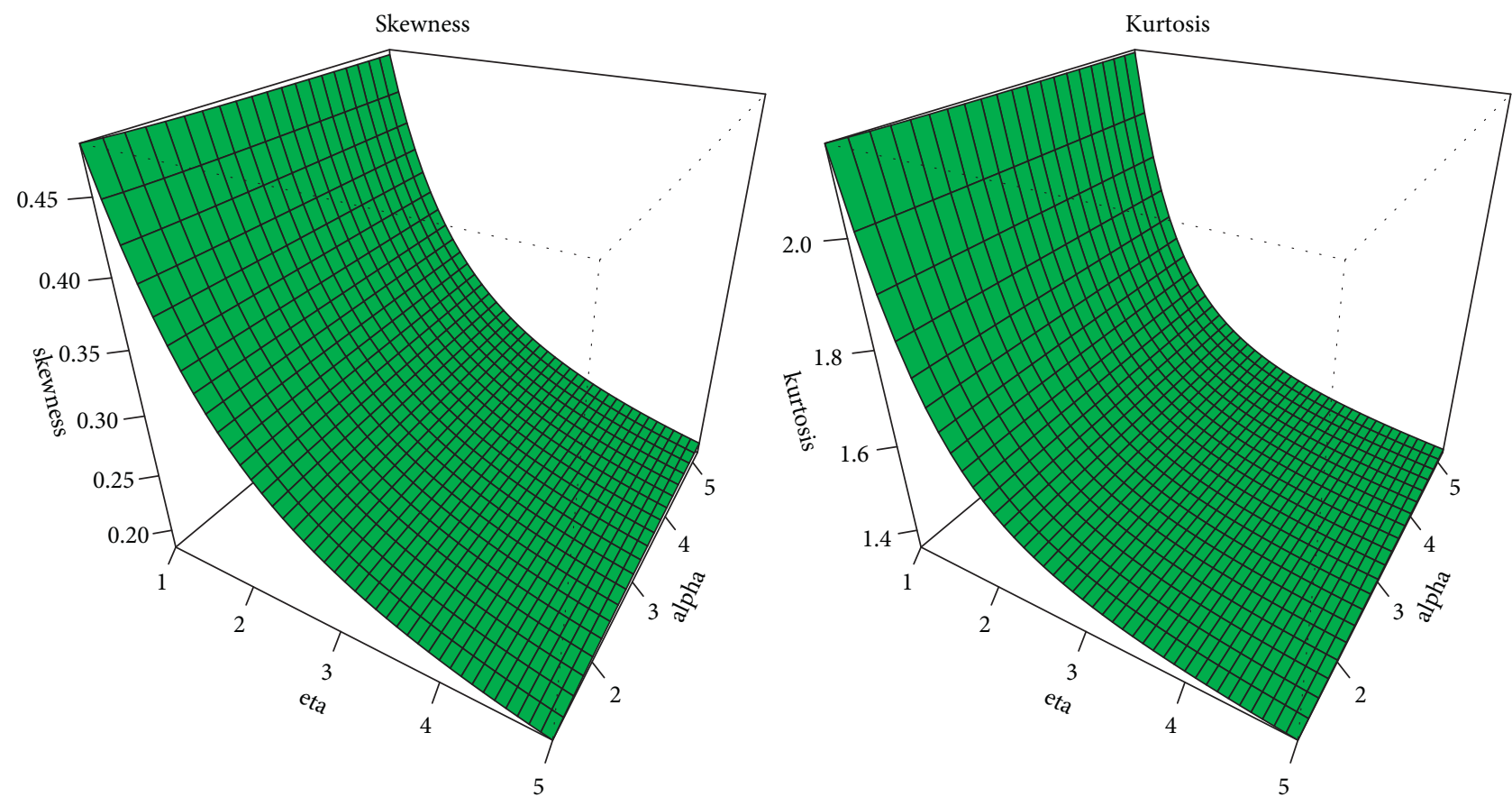

FIgURE 3: Plots of SK and KU of the IPBH distribution for several values of $\alpha$ and $\eta$.

$$
=\sum_{k=0}^{\infty} \frac{(-1)^{k} \alpha^{k} \pi[-\eta(\alpha-k)-r] \csc [\pi(k-(r / \eta))]}{k ! \eta} .
$$

The first four moments of the IPBH distribution follow from the above formula with $r=1,2,3$, and 4 .
The moment generating function of the IPBH distribution takes the following form:

$$
\begin{aligned}
M(t) & =\int_{0}^{\infty} e^{t x} f(x) \mathrm{d} x=\sum_{m, k=0}^{\infty} \frac{(-1)^{k} t^{m} \eta \alpha^{k}}{k ! m !} \int_{0}^{\infty} \frac{x^{m-\eta k-1}\left[\alpha+(\alpha+1) x^{\eta}\right]}{\left(x^{\eta}+1\right)^{2}} \mathrm{~d} x \\
& =\sum_{m, k=0}^{\infty} \frac{(-1)^{k} t^{m} \alpha^{k} \pi[-\eta(\alpha-k)-m] \csc [\pi(k-(m / \eta))]}{k ! m ! \eta} .
\end{aligned}
$$

3.3. Incomplete Moments. The $r$ th incomplete moment (ICM) of IPBH distribution follows as (for $k \eta<r$ )

$$
\begin{aligned}
\Psi_{r}(t) & =\int_{0}^{t} x^{r} f(x) \mathrm{d} x=\sum_{k=0}^{\infty} \frac{(-1)^{k} \eta \alpha^{k}}{k !} \int_{0}^{t} \frac{x^{r-\eta k-1}\left[\alpha+(\alpha+1) x^{\eta}\right]}{\left(x^{\eta}+1\right)^{2}} \mathrm{~d} x \\
& =\sum_{k=0}^{\infty} \frac{(-1)^{k} \alpha^{k}}{k ! \eta} t^{r-\eta k}\left\{\eta\left(\alpha+\frac{1}{t^{-\eta}+1}\right)+\left(-t^{\eta}\right)^{k-(r / \eta)}\left[\alpha D B_{-t^{\eta}}\left(\frac{r}{\eta}-k, 0\right)+(\alpha+1)(r-\eta k) B_{-t^{\eta}}\left(\frac{r}{\eta}-k+1,0\right)\right]\right\},
\end{aligned}
$$

where $D=(\eta+\eta k-r)$ and $B_{z}(a, b)=\int_{0}^{z} t^{a-1}(1-t)^{b-1} \mathrm{~d} t$.

The first ICM can be used to calculate the Bonferroni and Lorenz curves that are, respectively, defined by $L(p)=\left(\Psi_{1}(t) / \mu_{1}^{\prime}\right)$ and $B(p)=\left(\Psi_{1}\left(x_{p}\right) /\left(p \mu_{1}^{\prime}\right)\right)$, where $x_{p}$ can be determined numerically using equation (7) for a certain probability $p$. The two curves have their importance in insurance, economics, medicine, demography, and engineering. The first ICM is also adopted to calculate the mean 
residual life (MRL) and mean waiting time that are derived as $m_{1}(t)=\left(\left[1-\Psi_{1}(t)\right] /(S(t)-t)\right)$ and $M_{1}(t)=\left(\left(t-\Psi_{1}\right.\right.$ $(t)) / F(t))$, respectively.
3.4. Order Statistics. The density function of the $i$ th order statistic (OS) of the IPBH distribution takes the following form:

$$
\begin{aligned}
f_{i: n}(x) & =\frac{f(x)}{B(i, n-i+1)} \sum_{h=0}^{\infty}(-1)^{h}\left(\begin{array}{c}
n-i \\
h
\end{array}\right) F^{h+i-1}(x) \\
& =\frac{\eta n ! x^{-\eta-1}\left[\alpha+(\alpha+1) x^{\eta}\right]\left(x^{\eta} e^{-\alpha x^{-\eta}} / x^{\eta}+1\right)^{i}\left(1-\left(x^{\eta} e^{-\alpha x^{-\eta}} / x^{\eta}+1\right)\right)^{n-i}}{\Gamma(i)\left(x^{\eta}+1\right) \Gamma(-i+n+1)} .
\end{aligned}
$$

The associated CDF reduces to

$$
\begin{aligned}
F_{i: n}(x) & =\sum_{r=i}^{n}\left(\begin{array}{c}
n \\
r
\end{array}\right)(F(x))^{r}(1-F(x))^{n-r} \\
& =\left(\begin{array}{c}
n \\
i
\end{array}\right)\left(\frac{x^{\eta} e^{-\alpha x^{-\eta}}}{x^{\eta}+1}\right)^{i}\left(1-\frac{x^{\eta} e^{-\alpha x^{-\eta}}}{x^{\eta}+1}\right)^{n-i}{ }_{2} F_{1}\left[1, i-n ; i+1 ; \frac{-x^{\eta}}{\alpha e^{x^{-\eta}}\left(x^{\eta}+1\right)-x^{\eta}}\right],
\end{aligned}
$$

where ${ }_{2} F_{1}\left[1, i-n ; i+1 ;-\left(x^{\eta} /\left(\alpha e^{x^{-\eta}}\left(x^{\eta}+1\right)-x^{\eta}\right)\right)\right]$ is a hypergeometric function.

The PDFs and CDFs of the minimum OS, $\left(Y_{n}\right)$, and maximum OS, $\left(T_{n}\right)$, can be obtained simply from the last two formulae with $i=1$ and $i=n$, respectively. The limiting distributions of $\left(Y_{n}\right)$ and $\left(T_{n}\right)$ are expressed by Theorem 2.1.1 in [17].

$$
\begin{aligned}
& \lim _{n \longrightarrow+\infty} P\left(Y_{n}<K_{n} x\right)=\left\{\begin{array}{ll}
1, & 0<x<1, \\
1-e^{-1}, & x=1, \\
0, & x>1,
\end{array} \quad K_{n}=F^{-1}\left(\frac{1}{n}\right),\right. \\
& \lim _{n \longrightarrow+\infty} P\left(T_{n}<S_{n} x\right)=e^{x^{-\eta}}, \quad S_{n}=F^{-1}\left(1-\frac{1}{n}\right) \text {. } \\
& L(\varphi)=-\alpha \sum_{l=1}^{n} x_{l}^{-\eta}+\sum_{l=1}^{n} \log \left(\alpha+(\alpha+1) x_{l}^{\eta}\right) \\
& -2 \sum_{l=1}^{n} \log \left(x_{l}^{\eta}+1\right)-\sum_{l=1}^{n} \log \left(x_{l}\right)+n \log (\eta) .
\end{aligned}
$$

\section{Classical Inference}

In this section, different classical estimation methods of the IPBH parameters are discussed.

4.1. Maximum Likelihood. Consider the random sample of size $n$, say $x_{1}, x_{2}, \ldots, x_{n}$, from the PDF (4); then the loglikelihood function for $\varphi=(\alpha, \eta)^{T}, L(\varphi)$, reduces to
By differentiating equation (15) with respect to $\alpha$ and $\eta$, we get

$$
\begin{aligned}
& \frac{\partial L(\varphi)}{\partial \alpha}=\sum_{l=1}^{n} \frac{x_{l}^{\eta}+1}{\alpha+(\alpha+1) x_{l}^{\eta}}-\sum_{l=1}^{n} x_{l}^{-\eta} \\
& \frac{\partial L(\varphi)}{\partial \eta}=\alpha \sum_{l=1}^{n} x_{l}^{-\eta} \log \left(x_{l}\right)+\sum_{l=1}^{n} \frac{(\alpha+1) x_{l}^{\eta} \log \left(x_{l}\right)}{\alpha+(\alpha+1) x_{l}^{\eta}}-2 \sum_{l=1}^{n} \frac{x_{l}^{\eta} \log \left(x_{l}\right)}{x_{l}^{\eta}+1}+\frac{n}{\eta}
\end{aligned}
$$


Solving the three previous equations using the statistical software such as Maple, R, SAS, or Mathematica gives the MLE of the IPBH parameters.

4.2. Least Squares and Weighted Least Squares. Consider the order statistics of a random sample, say $x_{1: n}, x_{2: n}, \ldots, x_{n: n}$, from the IPBH distribution. Then, the LSE of the IPBH parameters follow by minimizing:

$$
\begin{aligned}
\operatorname{LS}(\varphi) & =\sum_{l=1}^{n}\left[F\left(x_{l: n}\right)-\frac{l}{n+1}\right]^{2} \\
& =\sum_{l=1}^{n}\left[1-\frac{l}{n+1}-\frac{e^{-\alpha x_{l: n}}}{x_{l: n}+1}\right]^{2} .
\end{aligned}
$$

We also can obtain the LSE by solving the formula

$$
\begin{aligned}
& \sum_{l=1}^{n}\left[F\left(x_{l: n}\right)-\frac{l}{n+1}\right]^{2} \\
& \quad=\sum_{l=1}^{n}\left[1-\frac{e^{-\alpha x_{l: n}}}{x_{l: n}+1}-\frac{l}{n+1}\right] \psi_{p}\left(x_{l: n}\right)=0, \quad p=1,2,
\end{aligned}
$$

where

$$
\begin{gathered}
\psi_{1}\left(x_{l: n}\right)=\frac{\partial}{\partial \alpha} F\left(x_{l: n}\right)=-\frac{e^{-\alpha x_{l: n}^{-\eta}}}{x_{l: n}^{\eta}+1} \\
\psi_{2}\left(x_{l: n}\right)=\frac{\partial}{\partial \eta} F\left(x_{l: n}\right)=\frac{\log \left(x_{l: n}\right) e^{-\alpha x_{l: n}^{-\eta}\left(\alpha+(\alpha+1) x_{l: n}^{\eta}\right)}}{\left(x_{l: n}^{\eta}+1\right)^{2}} .
\end{gathered}
$$

The WLSE of the IPBH parameters are calculated by minimizing:

$$
\begin{aligned}
W(\varphi) & =\sum_{l=1}^{n} \frac{(n+1)^{2}(n+2)}{l(n-l+1)}\left[F\left(x_{l: n}\right)-\frac{l}{n+1}\right]^{2} \\
& =\sum_{l=1}^{n} \frac{(n+1)^{2}(n+2)}{l(n-l+1)}\left[1-\frac{e^{-\alpha x_{l: n}}}{x_{l: n}+1}-\frac{l}{n+1}\right]^{2} .
\end{aligned}
$$

The WLSE are also calculated by solving the following formula:

$$
\sum_{l=1}^{n} \frac{(n+1)^{2}(n+2)}{l(n-l+1)}\left[1-\frac{e^{-\alpha x_{l: n}}}{x_{l: n}+1}-\frac{l}{n+1}\right] \psi_{p}\left(x_{l: n}\right)=0
$$

where $\psi_{p}\left(x_{l: n}\right), p=1,2$, are specified by (19) and (20).

4.3. Anderson-Darling and Right Tail Anderson-Darling. The ADE of the IPBH parameters are obtained by minimizing:

$$
\mathrm{AD}(\varphi)=-n-\frac{1}{n} \sum_{l=1}^{n}(2 l-1)\left[\log F\left(x_{l: n}\right)+\log S\left(x_{l: n}\right)\right]
$$

These estimators can be determined by the derivation of the following equation:

$$
\sum_{l=1}^{n}(2 l-1)\left[\frac{\psi_{p}\left(x_{l: n}\right)}{F\left(x_{l: n}\right)}-\frac{\psi_{p}\left(x_{n+1-l: n}\right)}{S\left(x_{n+1-l: n}\right)}\right]=0,
$$

where $\psi_{p}\left(x_{l: n}\right), p=1,2$, are specified by (19) and (20).

The RADE of the IPBH parameters can be determined by minimizing:

$$
\operatorname{RAD}(\varphi)=\frac{n}{2}-2 \sum_{l=1}^{n} F\left(x_{l: n}\right)-\frac{1}{n} \sum_{l=1}^{n}(2 l-1) \log \bar{F}\left(x_{n-l+1: n}\right)
$$

and they also can be determined by solving

$$
-2 \sum_{l=1}^{n} \psi_{p}\left(x_{l: n}\right)+\frac{1}{n} \sum_{l=1}^{n}(2 l-1) \frac{\psi_{p}\left(x_{n-l+1: n}\right)}{\bar{F}\left(x_{n-l+1: n}\right)}=0, \quad p=1,2
$$

where $\psi_{p}\left(x_{l: n}\right), p=1,2$, are specified by (19) and (20).

4.4. Cramér-von Mises. The CVME of the IPBH parameters are derived by minimizing:

$$
\begin{aligned}
\mathrm{CV}(\varphi) & =\frac{1}{12 n}+\sum_{l=1}^{n}\left[F\left(x_{l: n}\right)-\frac{2 l-1}{2 n}\right]^{2} \\
& =\frac{1}{12 n}+\sum_{l=1}^{n}\left[1-\frac{e^{-\alpha x_{l: n}}}{x_{l: n}+1}-\frac{2 l-1}{2 n}\right]^{2}
\end{aligned}
$$

The CVME can also be derived by solving the following formula:

$$
\sum_{l=1}^{n}\left[1-\frac{e^{-\alpha x_{l: n}}}{x_{l: n}+1}-\frac{2 l-1}{2 n}\right] \psi_{p}\left(x_{l: n}\right)=0,
$$

where $\psi_{p}\left(x_{l: n}\right), p=1,2$, are specified by (19) and (20).

4.5. Maximum Product of Spacings. The maximum product of spacings (MPS) approach is a useful alternative to the ML approach. The uniform spacings, say $D_{l}$, of a random sample from the IPBH distribution are defined by

$$
D_{l}=F\left(x_{l}\right)-F\left(x_{l-1}\right)
$$

where $\sum_{l=1}^{n+1} D_{l}=1, F\left(x_{0}\right)=0$, and $F\left(x_{n+1}\right)=1$. The MPSE of the IPBH parameters are obtained by maximizing:

$$
\operatorname{MP}(\varphi)=\frac{1}{n+1} \sum_{l=1}^{n+1} \log \left(D_{l}\right)
$$

Moreover, the MPSE can be determined using 


$$
\frac{1}{n+1} \sum_{l=1}^{n+1} \frac{1}{D_{l}}\left[\psi_{p}\left(x_{l: n}\right)-\psi_{p}\left(x_{l-1: n}\right)\right]=0,
$$

where $\psi_{p}\left(x_{l: n}\right), p=1,2,3$, are specified by (19) and (20).

\section{Bayesian Estimation}

In this section, we estimate the parameters of the IPBH distribution from complete sample by the Bayes estimators (BE) using symmetric and asymmetric loss functions. Now, we adopted the SE, GE, and LN loss functions to obtain the parameters estimates. We also consider that $\alpha$ and $\eta$ are independent. We adopted two independent gamma priors for the two parameters $\alpha$ and $\eta$.

The two independent gamma priors have the forms

$$
\begin{aligned}
& \pi_{1}(\alpha) \propto \alpha^{\mu_{1}-1} e^{-\alpha \lambda_{1},}, \\
& \pi_{2}(\eta) \propto \eta^{\mu_{2}-1} e^{-\eta \lambda_{2},}
\end{aligned}
$$

respectively, where $\mu_{1}, \mu_{2}, \lambda_{1}, \lambda_{2}>0$.

Then, the joint PDF prior of $\alpha$ and $\eta$ takes the form

$$
\pi(\alpha, \eta) \propto \alpha^{\mu_{1}-1} \eta^{\mu_{2}-1} e^{-\left(\alpha \lambda_{1}+\eta \lambda_{2}\right)} .
$$

Hence, the posterior function reduces to

$$
\pi^{*}(\alpha, \eta) \propto \alpha^{\mu_{1}-1} \eta^{n+\mu_{2}-1} e^{-\left(\alpha \lambda_{1}+\eta \lambda_{2}\right)} e_{i=1}^{-\alpha} \sum_{n x_{i}^{-\eta}} \prod_{i=1}^{n} \frac{\alpha+(\alpha+1) x_{i}^{\eta}}{x_{i}\left(x_{i}^{\eta}+1\right)^{2}} .
$$

According to the SE loss function, the $\mathrm{BE}$ for $B=B(\Theta)$, $\Theta=(\alpha, \eta)$, is

$$
\widehat{B}_{\mathrm{SE}}=\int_{\Theta} B \pi^{*}(\Theta) \mathrm{d} \Theta,
$$

where $\pi^{*}(\Theta)$ is as in equation (34). The BE under the LN loss function has the form

$$
\widehat{B}_{\mathrm{LN}}=-\frac{1}{c} \log \left(E_{\Theta}[\exp (-c \Theta)]\right),
$$

such that $E_{\Theta}[\exp (-c \Theta)]$ exists. The $\mathrm{BE} \widehat{\Theta}_{\mathrm{GE}}$ under GE loss function is

$$
\widehat{B}_{\mathrm{GE}}=\left(E_{\Theta}\left[\Theta^{-q}\right]\right)^{-(1 / q)},
$$

such that $E_{\Theta}\left[\Theta^{-q}\right]$ exists. In fact, the integrals in equations (35)-(37) cannot be found analytically. Hence, the Markov chain Monte Carlo (MCMC) technique is adopted to approximate these integrals. Moreover, we use the MetropolisHastings algorithm as an example of the MCMC technique to obtain the estimates.

\section{Simulation Results}

This section is devoted to determining the performance and behavior of several estimation approaches in estimating the IPBH parameters based on detailed simulation results. For this purpose, several sample sizes, $n=\{20,50,100,200,500\}$, and several values of the parameters $\alpha$ and $\eta$, $\alpha=0.5,0.75,1.5$ and $\eta=0.5,1.5$, are considered. We generated $N=5000$ random samples from the IPBH distribution using its QF (6). The compared estimators are checked in terms of their average absolute biases (BIAS), average mean square errors (MSE), and average mean relative errors of the estimates (MRE) which are obtained, for all parameter values and sample sizes, using the R program.

The BIAS, MSE, and MRE can be determined by the three following equations:

$$
\begin{aligned}
& \text { BIAS }=\frac{1}{N} \sum_{i=1}^{N}|\widehat{\boldsymbol{\theta}}-\boldsymbol{\theta}|, \\
& \text { MSE }=\frac{1}{N} \sum_{i=1}^{N}(\widehat{\boldsymbol{\theta}}-\boldsymbol{\theta})^{2}, \\
& \text { MRE }=\frac{1}{N} \sum_{i=1}^{N} \frac{|\widehat{\boldsymbol{\theta}}-\boldsymbol{\theta}|}{\boldsymbol{\theta}},
\end{aligned}
$$

where $\theta=(\alpha, \eta)$.

Tables 1-4 report the simulation results including BIAS, MSE, and MRE of the IPBH parameters using the ten estimation approaches. Moreover, Tables 1-4 report the rank of each one of the ten estimators among all the estimators in each row by the superscript indicators, and the partial sum of the ranks for each column, say $\sum$ Ranks, in a certain sample size. From the tabulated results, it is observed that the ten estimation methods show the property of consistency for all studied cases.

Table 5 displays the partial and overall rank of these estimators. Form the results in Table 5, we can conclude that the Bayesian method outperforms all other classical methods under the three loss functions, with respective overall scores of 29,46 , and 50 for the SE, GE, and LN loss functions, respectively. Therefore, we confirm the superiority of the Bayesian approach for the IPBH distribution.

\section{Two Real-Life Applications}

This section is devoted to analyzing two real-life datasets to explore the importance and flexibility of the IPBH distribution as compared with some of its other competing distributions.

7.1. Dataset I. This dataset consists of 63 observations which are generated to simulate the strengths of glass fibers [18]. The 63 observations of the dataset are as follows: 1.014, 1.081, $1.082,1.185,1.223,1.248,1.267,1.271,1.272,1.275,1.276$, $1.278,1.286,1.288,1.292,1.304,1.306,1.355,1.361,1.364$, $1.379,1.409,1.426,1.459,1.460,1.476,1.481,1.484,1.501$, $1.506,1.524,1.526,1.535,1.541,1.568,1.579,1.581,1.591$, $1.593,1.602,1.666,1.670,1.684,1.691,1.704,1.731,1.735$, $1.747,1.748,1.757,1.800,1.806,1.867,1.876,1.878,1.910$, $1.916,1.972,2.012,2.456,2.592,3.197$, and 4.121 .

7.2. Dataset II. This dataset represents the relief times of 20 patients who are receiving an analgesic [19]. The 20 relief times are as follows: 1.1, 1.4 1.3, 1.7, 1.9, 1.8, 1.6, 2.2, 1.7, 2.7, $4.1,1.8,1.5,1.2,1.4,3,1.7,2.3,1.6$, and 2 . 


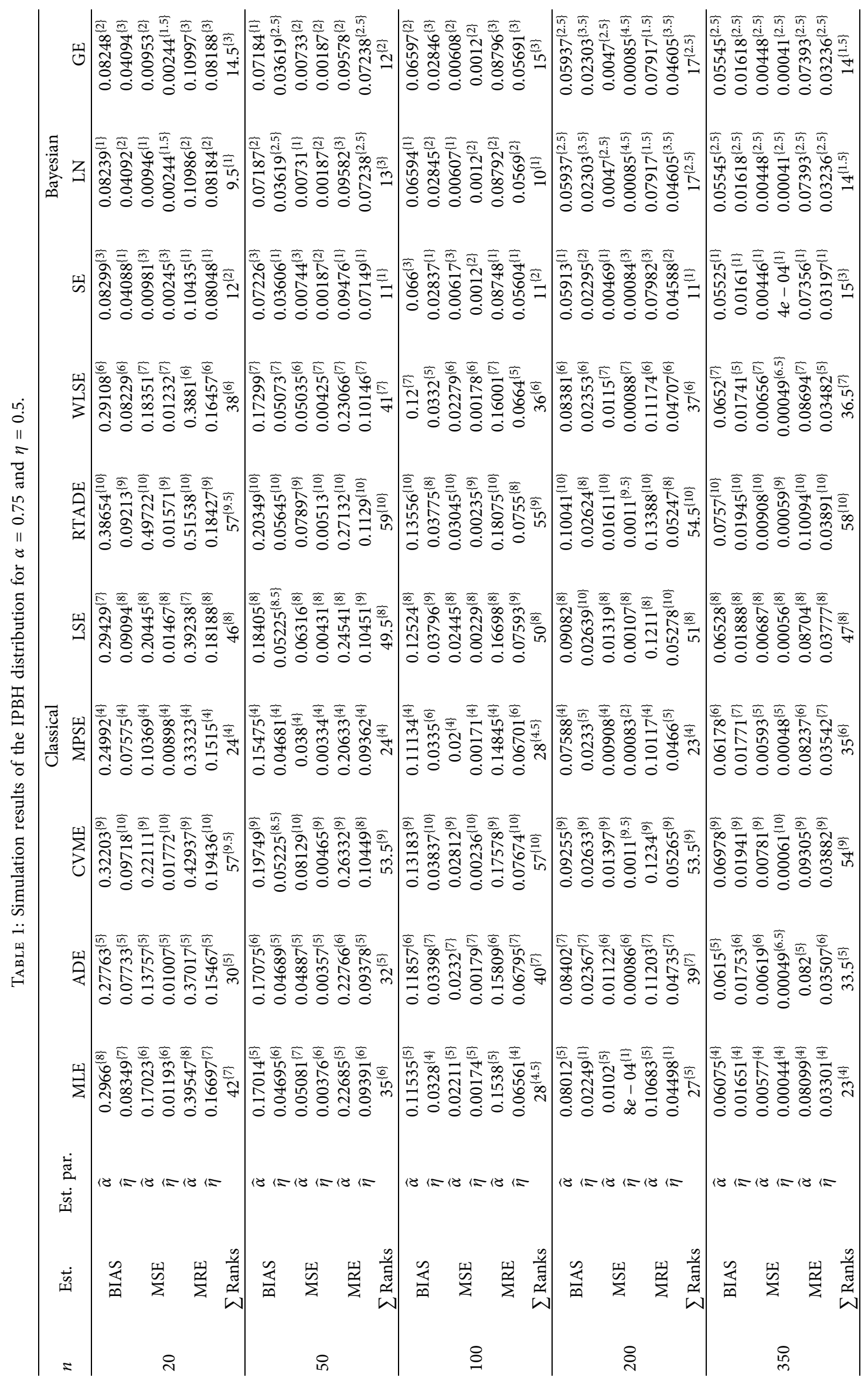




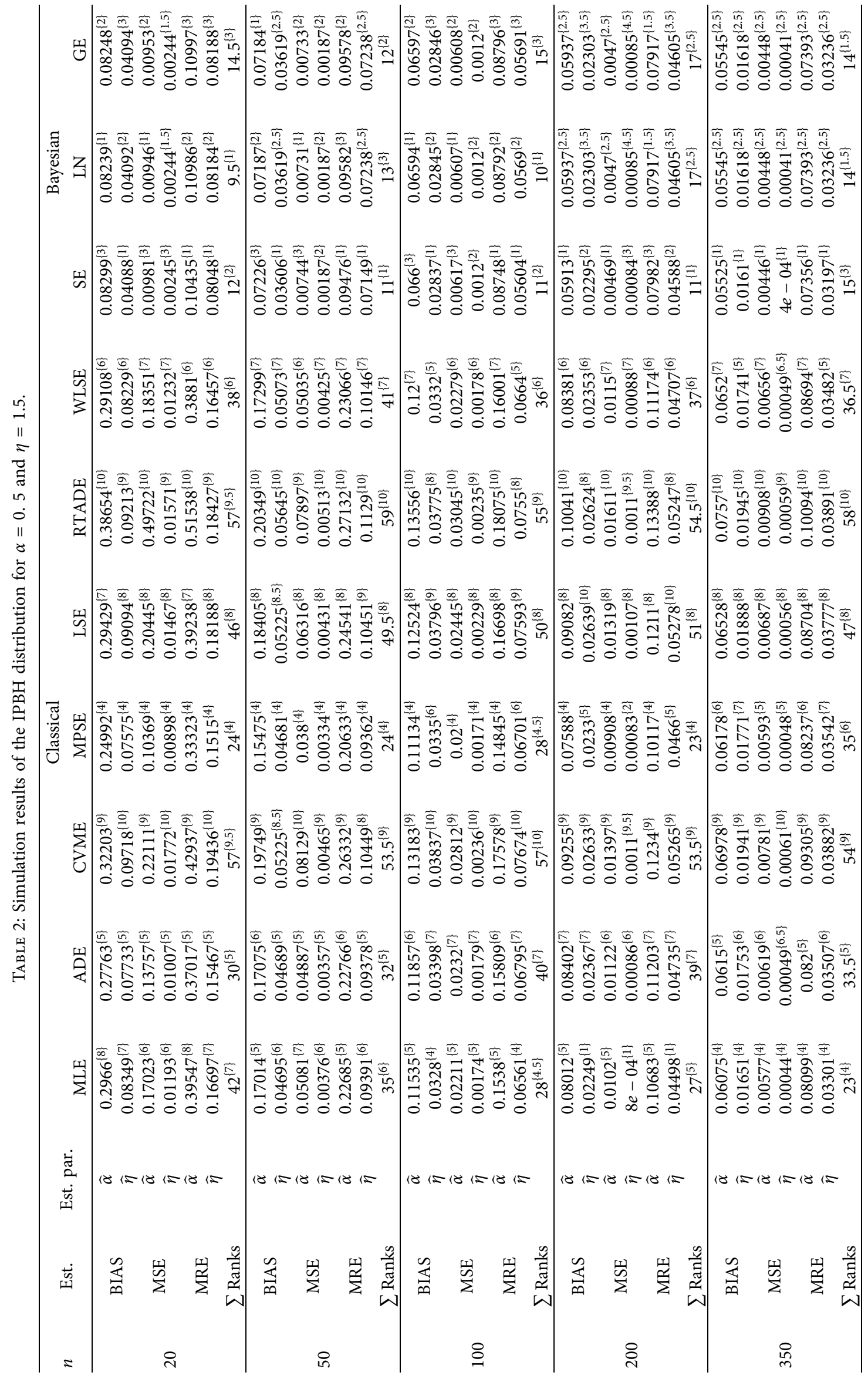




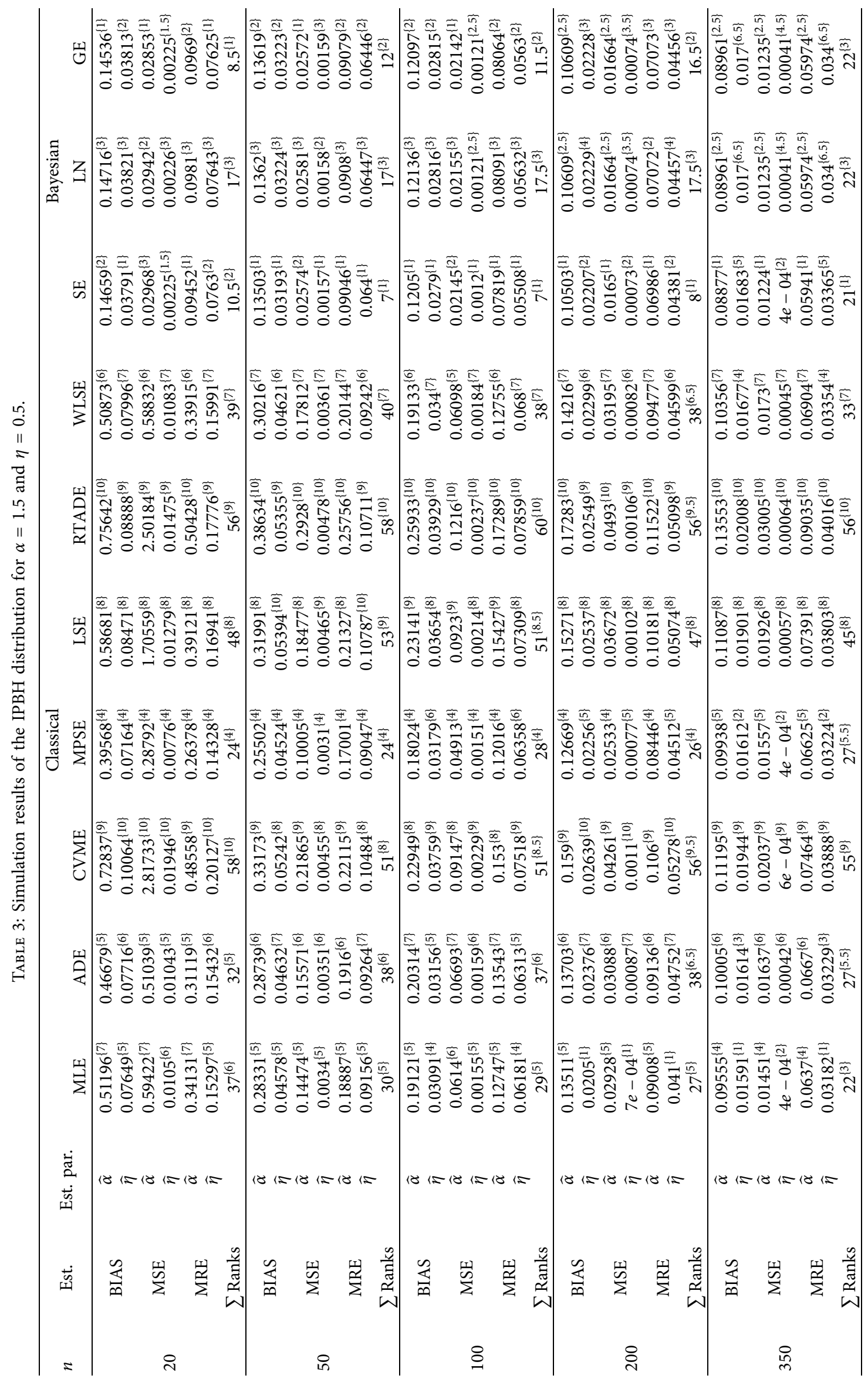




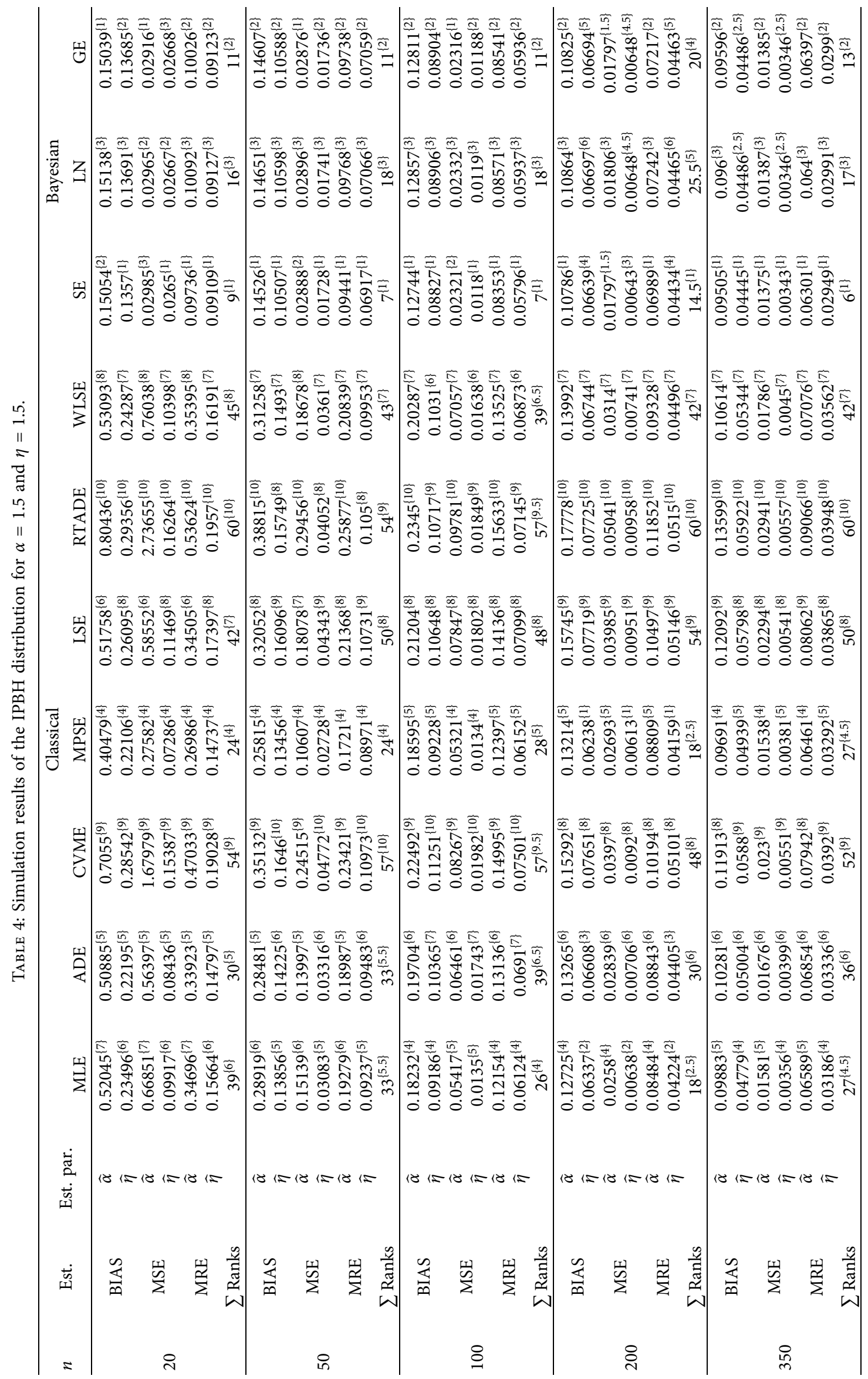


TABLE 5: Partial and overall ranks of the ten estimation methods for the IPBH distribution.

\begin{tabular}{|c|c|c|c|c|c|c|c|c|c|c|c|}
\hline \multirow{2}{*}{ Parameter } & \multirow{2}{*}{$n$} & \multicolumn{7}{|c|}{ Classical } & \multicolumn{3}{|c|}{ Bayesian } \\
\hline & & MLE & $\mathrm{ADE}$ & CVME & MPSE & LSE & RTADE & WLSE & SE & $\mathrm{LN}$ & GE \\
\hline \multirow{5}{*}{$\alpha=0.725, \eta=0.5$} & 20 & 7 & 5 & 9.5 & 4 & 8 & 9.5 & 6 & 2 & 1 & 3 \\
\hline & 50 & 6 & 5 & 9 & 4 & 8 & 10 & 7 & 1 & 3 & 2 \\
\hline & 100 & 4.5 & 7 & 10 & 4.5 & 8 & 9 & 6 & 2 & 1 & 3 \\
\hline & 200 & 5 & 7 & 9 & 4 & 8 & 10 & 6 & 1 & 2.5 & 2.5 \\
\hline & 350 & 4 & 5 & 9 & 6 & 8 & 10 & 7 & 3 & 1.5 & 1.5 \\
\hline \multirow{5}{*}{$\alpha=0.5, \eta=1.5$} & 20 & 7 & 5 & 9.5 & 4 & 8 & 9.5 & 6 & 2 & 1 & 3 \\
\hline & 50 & 6 & 5 & 9 & 4 & 8 & 10 & 7 & 1 & 3 & 2 \\
\hline & 100 & 4.5 & 7 & 10 & 4.5 & 8 & 9 & 6 & 2 & 1 & 3 \\
\hline & 200 & 5 & 7 & 9 & 4 & 8 & 10 & 6 & 1 & 2.5 & 2.5 \\
\hline & 350 & 4 & 5 & 9 & 6 & 8 & 10 & 7 & 3 & 1.5 & 1.5 \\
\hline \multirow{5}{*}{$\alpha=1.5, \eta=0.5$} & 20 & 6 & 5 & 10 & 4 & 8 & 9 & 7 & 2 & 3 & 1 \\
\hline & 50 & 5 & 6 & 8 & 4 & 9 & 10 & 7 & 1 & 3 & 2 \\
\hline & 100 & 5 & 6 & 8.5 & 4 & 8.5 & 10 & 7 & 1 & 3 & 2 \\
\hline & 200 & 5 & 6.5 & 9.5 & 4 & 8 & 9.5 & 6.5 & 1 & 3 & 2 \\
\hline & 350 & 3 & 5.5 & 9 & 5.5 & 8 & 10 & 7 & 1 & 3 & 3 \\
\hline \multirow{5}{*}{$\alpha=1.5, \eta=1.5$} & 20 & 6 & 5 & 9 & 4 & 7 & 10 & 8 & 1 & 3 & 2 \\
\hline & 50 & 5.5 & 5.5 & 10 & 4 & 8 & 9 & 7 & 1 & 3 & 2 \\
\hline & 100 & 4 & 6.5 & 9.5 & 5 & 8 & 9.5 & 6.5 & 1 & 3 & 2 \\
\hline & 200 & 2.5 & 6 & 8 & 2.5 & 9 & 10 & 7 & 1 & 5 & 4 \\
\hline & 350 & 4.5 & 6 & 9 & 4.5 & 8 & 10 & 7 & 1 & 3 & 2 \\
\hline$\sum$ Ranks & & 99.5 & 116 & 183.5 & 86.5 & 161.5 & 194 & 134 & 29 & 50 & 46 \\
\hline Overall rank & & 5 & 6 & 9 & 4 & 8 & 10 & 7 & 1 & 3 & 2 \\
\hline
\end{tabular}

TABLE 6: The estimates of the parameters of the IPBH distribution and other competing models with several discrimination statistics for dataset I.

\begin{tabular}{|c|c|c|c|c|c|c|c|c|c|c|c|}
\hline Distribution & Estimates & SEs & $-\widehat{\ell}$ & AIC & CAIC & BIC & HQIC & $W$ & $A$ & K-S (stat) & $\mathrm{K}-\mathrm{S} p$ value \\
\hline \multirow{2}{*}{ IPBH } & $\widehat{\alpha}=5.7160$ & 1.2042 & 20.0086 & 44.0172 & 44.2172 & 48.3035 & 45.7030 & 0.5175 & 0.0681 & 0.0762 & 0.8573 \\
\hline & $\widehat{\eta}=5.4950$ & 0.5120 & & & & & & & & & \\
\hline $\mathrm{BH}$ & $\hat{a}=0.2325$ & 0.0776 & 113.364 & 228.729 & 228.794 & 230.872 & 229.571 & 30.1567 & 6.7363 & 0.6103 & $<0.001$ \\
\hline \multirow{2}{*}{$W$} & $\widehat{a}=3.0620$ & 0.2403 & 46.3669 & 96.7338 & 96.9338 & 101.02 & 98.4196 & 5.2608 & 0.8853 & 0.2051 & 0.0099 \\
\hline & $\widehat{b}=1.7875$ & 0.0784 & & & & & & & & & \\
\hline $\mathrm{E}$ & $\widehat{a}=0.6189$ & 0.0779 & 93.2229 & 188.446 & 188.511 & 190.589 & 189.289 & 18.003 & 3.8455 & 0.4721 & $<0.001$ \\
\hline ILL & $\widehat{\alpha}=3.2980$ & 0.3330 & 76.8 & 155.6 & 155.666 & 157.743 & 156.443 & 35.3833 & 7.6890 & 0.5966 & $<0.001$ \\
\hline \multirow{2}{*}{$F$} & $\widehat{\alpha}=5.4378$ & 0.5192 & 20.0639 & 44.1277 & 44.3277 & 48.414 & 45.8135 & 0.5290 & 0.0698 & 0.0772 & 0.8466 \\
\hline & $\widehat{\lambda}=1.4108$ & 0.0344 & & & & & & & & & \\
\hline \multirow{2}{*}{ G } & $\widehat{\alpha}=15.8624$ & 2.7970 & 31.2066 & 66.4132 & 66.6132 & 70.6995 & 68.099 & 2.0668 & 0.3014 & 0.1301 & 0.2362 \\
\hline & $\widehat{\lambda}=0.1018$ & 0.0182 & & & & & & & & & \\
\hline \multirow{2}{*}{ IWL } & $\widehat{\alpha}=19.8452$ & 3.5326 & 23.7532 & 51.5064 & 51.7064 & 55.7927 & 53.1922 & 0.9444 & 0.1201 & 0.0880 & 0.7130 \\
\hline & $\widehat{\lambda}=30.8811$ & 5.4602 & & & & & & & & & \\
\hline IL & $\widehat{\lambda}=2.0297$ & 0.2053 & 89.3345 & 180.669 & 180.735 & 182.812 & 181.512 & 17.3589 & 3.6202 & 0.4504 & $<0.001$ \\
\hline IP & $\begin{array}{l}\widehat{\alpha}=120388 \\
\widehat{\theta}=0.00001\end{array}$ & $\begin{array}{c}1.651 \times 10^{6} \\
0.0017\end{array}$ & 92.805 & 189.61 & 189.81 & 193.896 & 191.296 & 18.5169 & 3.9072 & 0.4681 & $<0.001$ \\
\hline \multirow{2}{*}{ INM } & $\widehat{\alpha}=5.5617$ & 0.9627 & 21.7736 & 47.5472 & 47.7472 & 51.8335 & 49.233 & 0.6841 & 0.0834 & 0.0793 & 0.8222 \\
\hline & $\widehat{\lambda}=0.4479$ & 0.0239 & & & & & & & & & \\
\hline
\end{tabular}

Here, we show empirically that the IPBH distribution can provide a more adequate fit than ten competing distributions, namely, the BH, W, E, ILL, F, G, IWL, IL, IP, and INM distributions. We adopted some discrimination or information criterions (IC) such as minus maximized loglikelihood $(-\widehat{\ell})$, Akaike IC (AIC), the corrected AIC (CAIC), Hannan-Quinn IC (HQIC), Bayesian IC (BIC), AndersonDarling $(A)$, Cramér-von Mises $(W)$, and KolmogorovSmirnov (K-S) statistics and $p$ value (K-S $p$ value) to check the studied competing distributions.
Tables 6 and 7 report estimates of the parameters, by the maximum likelihood approach, standard errors (SEs), and the nine discrimination measures for the two datasets, respectively. The figures in these tables show that the new IPBH model provides a close fit to both modeled datasets among other competing distributions. The fitted curves for the PDF, CDF, SF, and P-P plots of the IPBH distribution are depicted in Figures 4 and 5 for the two datasets, respectively. The values of discrimination measures in Tables 6 and 7 show great improvement in fitting using the IPBH model 
TABLE 7: The estimates of the parameters of the IPBH distribution and other competing models with several discrimination statistics for dataset II.

\begin{tabular}{|c|c|c|c|c|c|c|c|c|c|c|c|}
\hline Distribution & Estimates & SEs & $-\widehat{\ell}$ & AIC & CAIC & $\mathrm{BIC}$ & HQIC & $W$ & $A$ & K-S (stat) & K-S $p$ value \\
\hline \multirow{2}{*}{ IPBH } & $\widehat{\alpha}=5.2423$ & 1.9529 & 15.4046 & 34.8092 & 35.5151 & 36.8007 & 35.198 & 0.1528 & 0.0261 & 0.1005 & 0.9875 \\
\hline & $\widehat{\eta}=4.0622$ & 0.6871 & & & & & & & & & \\
\hline $\mathrm{BH}$ & $\widehat{a}=0.1756$ & 0.1165 & 40.1492 & 82.2985 & 82.5207 & 83.2942 & 82.4929 & 9.2419 & 2.0722 & 0.6074 & $<0.001$ \\
\hline \multirow{2}{*}{$W$} & $\widehat{a}=2.7870$ & 0.4273 & 20.5864 & 45.1728 & 45.8787 & 47.1643 & 45.5616 & 1.0835 & 0.1834 & 0.1849 & 0.5005 \\
\hline & $\widehat{b}=2.1299$ & 0.1820 & & & & & & & & & \\
\hline$E$ & $\widehat{a}=0.5263$ & 0.1176 & 32.8371 & 67.6742 & 67.8964 & 68.6699 & 67.8685 & 4.6035 & 0.9629 & 0.4395 & 0.0008 \\
\hline ILL & $\widehat{\alpha}=2.4916$ & 0.4479 & 32.8025 & 67.605 & 67.8272 & 68.6007 & 67.7993 & 10.8651 & 2.3617 & 0.5616 & $<0.001$ \\
\hline \multirow{2}{*}{$F$} & $\widehat{\alpha}=4.0174$ & 0.6972 & 15.4087 & 34.8174 & 35.5233 & 36.8089 & 35.2062 & 0.1545 & 0.0265 & 0.1019 & 0.9854 \\
\hline & $\widehat{\lambda}=1.5634$ & 0.0917 & & & & & & & & & \\
\hline \multirow{2}{*}{ G } & $\widehat{\alpha}=9.6694$ & 3.0064 & 17.8186 & 39.6372 & 40.3431 & 41.6287 & 40.0259 & 0.5990 & 0.1025 & 0.1734 & 0.5844 \\
\hline & $\widehat{\lambda}=0.1964$ & 0.0627 & & & & & & & & & \\
\hline \multirow{2}{*}{ IWL } & $\widehat{\alpha}=11.3572$ & 3.5778 & 16.0382 & 36.0764 & 36.7823 & 38.0679 & 36.4652 & 0.2694 & 0.0460 & 0.1318 & 0.8776 \\
\hline & $\widehat{\lambda}=20.2088$ & 6.3114 & & & & & & & & & \\
\hline IL & $\widehat{\lambda}=2.2546$ & 0.4089 & 31.7572 & 65.5144 & 65.7366 & 66.5101 & 65.7088 & 4.4688 & 0.9054 & 0.3694 & 0.0085 \\
\hline IP & $\begin{array}{l}\widehat{\alpha}=139428 \\
\widehat{\theta}=0.00001\end{array}$ & $\begin{array}{c}4.18528 \times 10^{6} \\
0.0003\end{array}$ & 32.6687 & 69.3375 & 70.0434 & 71.3289 & 69.7262 & 4.8020 & 0.9872 & 0.3872 & 0.0049 \\
\hline \multirow{2}{*}{ INM } & $\widehat{\alpha}=3.2242$ & 0.9714 & 15.6214 & 35.2428 & 35.9486 & 37.2342 & 35.6315 & 0.1919 & 0.0329 & 0.1138 & 0.9579 \\
\hline & $\widehat{\lambda}=0.3622$ & 0.0451 & & & & & & & & & \\
\hline
\end{tabular}
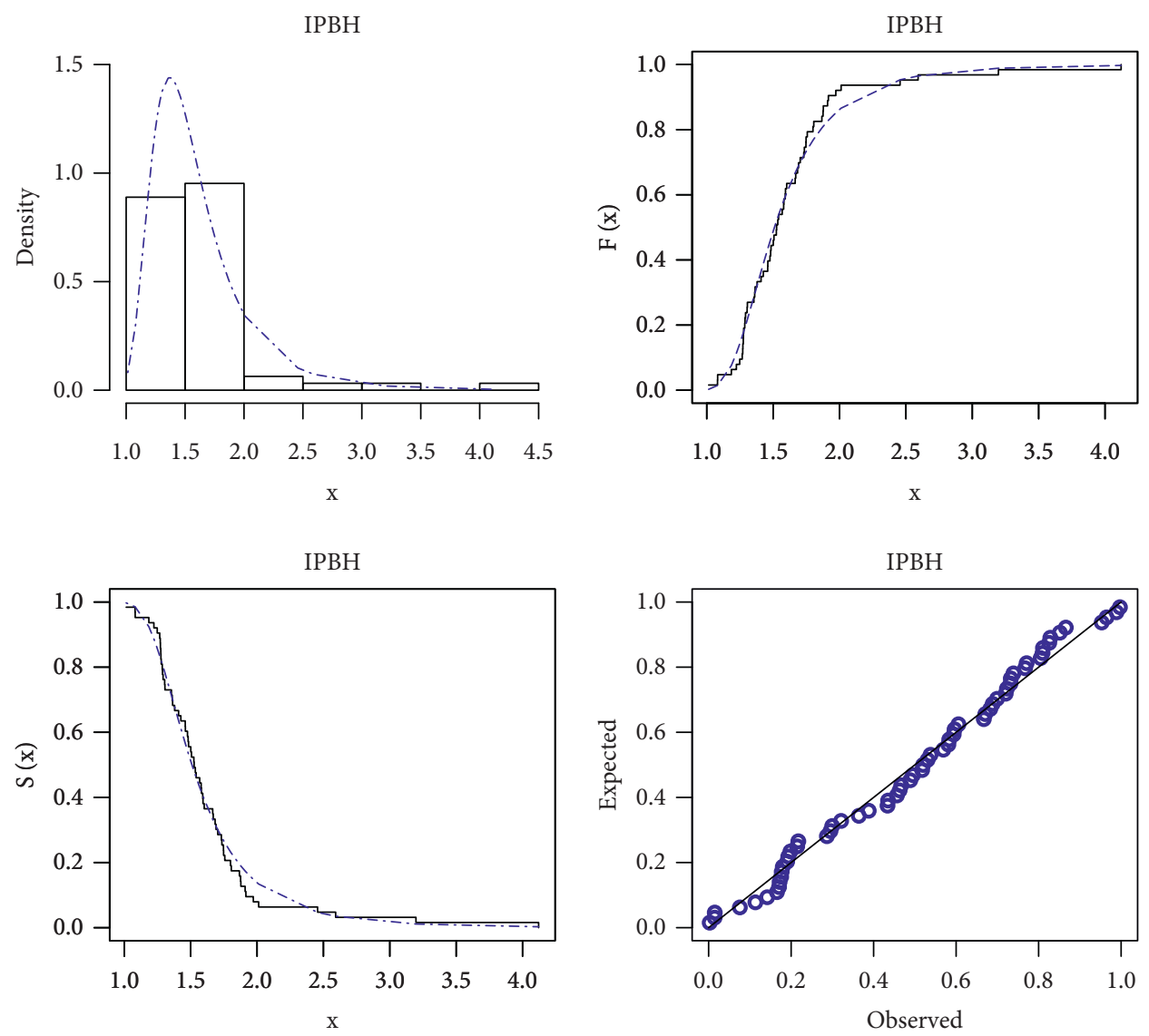

FIGURE 4: Histogram of dataset I with the fitted IPBH PDF, CDF, SF, and P-P plot.

over the $\mathrm{BH}$ model. For dataset $\mathrm{I}$, the K-S $p$ value of the IPBH distribution is 0.8573 , whereas it is less than 0.00001 for the $\mathrm{BH}$ model. Further, for dataset II, the K-S $p$ value of the $\mathrm{BH}$ distribution is also less than 0.00001 for the $\mathrm{BH}$ model, while it grows to be 0.9875 for the IPBH distribution.
The ten estimation approaches are also adopted to estimate the IPBH parameters from the two datasets. Tables 8 and 9 report the estimates of $\alpha$ and $\eta$ along with the values of $-\widehat{\ell}, A, W, \mathrm{~K}-\mathrm{S}$, and K-S $p$ value for both datasets, respectively. The proposed estimation approaches show a similar 
IPBH
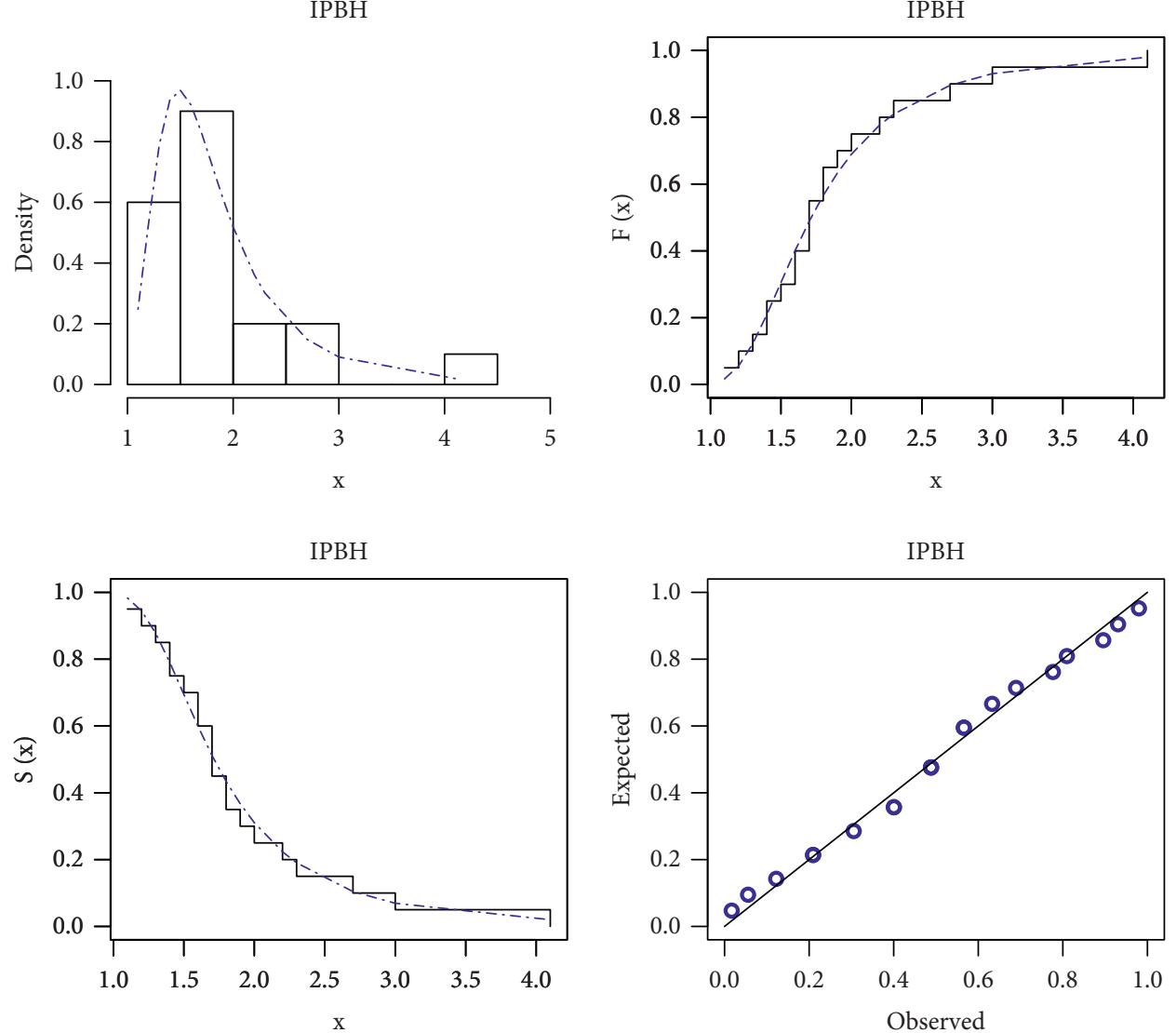

Figure 5: Histogram of dataset II with the fitted IPBH PDF, CDF, SF, and P-P plot.

TABLE 8: The estimates of $\alpha, \eta,-\widehat{\ell}, A, W, \mathrm{~K}-\mathrm{S}$ (stat), and K-S $p$ value of the IPBH distribution for dataset I.

\begin{tabular}{lccccccc}
\hline & $\hat{\alpha}$ & $\hat{\eta}$ & $-\hat{\ell}$ & $A$ & $W$ & K-S (stat) & K-S $p$ value \\
\hline MLE & 5.7160 & 5.4950 & 20.0086 & 0.5175 & 0.0680 & 0.0762 & 0.8573 \\
ADE & 6.4795 & 5.7664 & 20.1976 & 0.4776 & 0.0568 & 0.0662 & 0.9452 \\
CVME & 7.1013 & 5.9308 & 20.5657 & 0.5025 & 0.0539 & 0.0767 & 0.0767 \\
MPSE & 4.9180 & 5.1487 & 20.5657 & 0.5025 & 0.0539 & 0.0710 & 0.8520 \\
LSE & 6.7066 & 5.8074 & 20.3068 & 0.4845 & 0.0548 & 0.9084 \\
RTADE & 7.5598 & 6.0797 & 20.9502 & 0.5391 & 0.0552 & 0.0718 \\
WLSE & 6.9539 & 5.9190 & 20.4733 & 0.4903 & 0.0548 & 0.0808 \\
BSE & 5.8139 & 5.4722 & 20.0231 & 0.5246 & 0.0674 & 0.08013 \\
BLN & 5.8148 & 5.4729 & 20.0230 & 0.5243 & 0.0673 & 0.8043 \\
BGE & 5.8136 & 5.4719 & 20.0232 & 0.5247 & 0.0674 & 0.0808 & 0.8050 \\
\hline
\end{tabular}

TABLE 9: The estimates of $\alpha, \eta,-\hat{\ell}, A, W, \mathrm{~K}-\mathrm{S}$ (stat), and K-S $p$ value of the IPBH distribution for dataset II.

\begin{tabular}{lccccccc}
\hline & $\widehat{\alpha}$ & $\hat{\eta}$ & $-\widehat{\ell}$ & $A$ & $W$ & K-S (stat) & K-S $p$ value \\
\hline MLE & 5.2423 & 4.0622 & 15.4046 & 0.1528 & 0.0261 & 0.1005 & 0.9875 \\
ADE & 5.1651 & 4.0264 & 15.406 & 0.1522 & 0.0265 & 0.0990 & 0.9895 \\
CVME & 6.0804 & 4.2881 & 15.4871 & 0.1770 & 0.0244 & 0.0924 & 0.0924 \\
MPSE & 6.3782 & 4.8187 & 15.4871 & 0.1770 & 0.0244 & 0.0999 & 0.9955 \\
LSE & 4.9267 & 3.9463 & 15.4211 & 0.1544 & 0.0279 & 0.9882 \\
RTADE & 5.4326 & 4.1085 & 15.4092 & 0.1546 & 0.0254 & 0.0972 & 0.9915 \\
WLSE & 4.3142 & 3.7311 & 15.5535 & 0.1830 & 0.0342 & 0.1038 & 0.9977 \\
BSE & 5.4296 & 4.0412 & 15.4190 & 0.1627 & 0.0273 & 0.0980 & 0.9906 \\
BLN & 5.4366 & 4.0416 & 15.4198 & 0.1632 & 0.0273 & 0.0976 & 0.9911 \\
BGE & 5.4267 & 4.0410 & 15.4188 & 0.1625 & 0.0272 & 0.007 \\
\hline
\end{tabular}




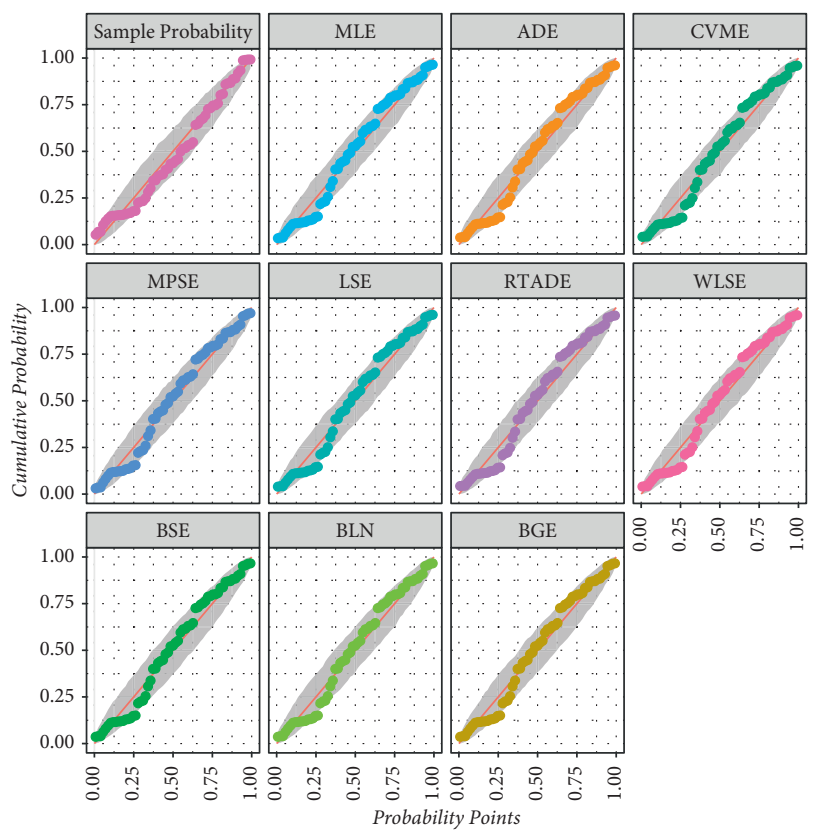

(a)

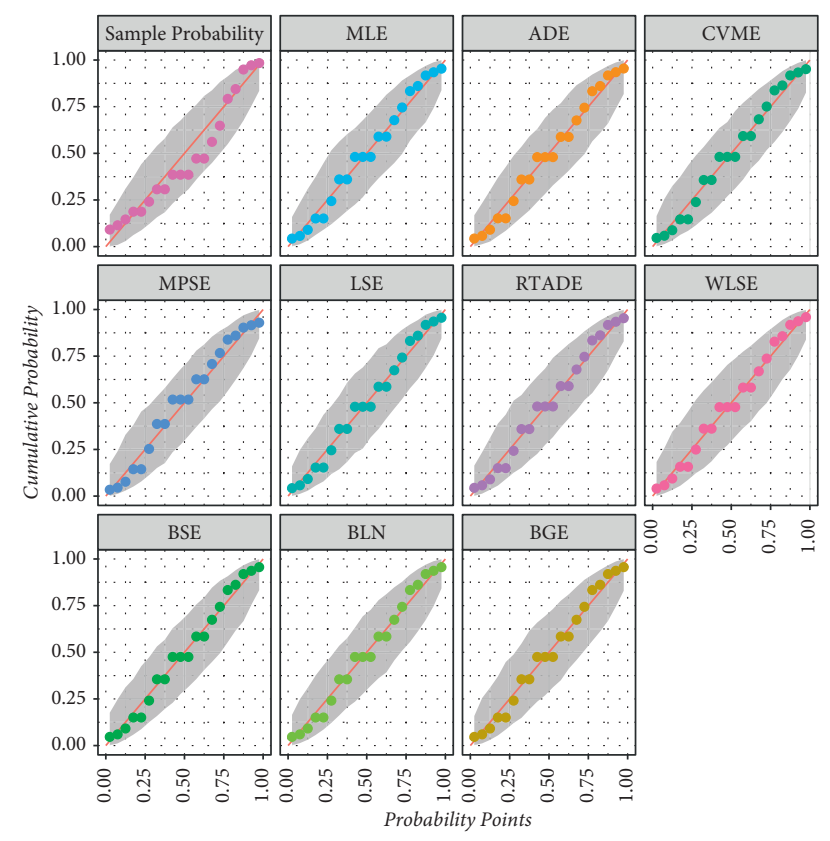

(b)

FIgURe 6: P-P plots of the IPBH distribution using several estimation approaches for dataset I (a) and dataset II (b).

very well performance in estimating $\alpha$ and $\eta$ of the IPBH distribution. A visual comparison shows the close performance of these estimators as shown in Figure 6 which represents the P-P plots of the IPBH distribution for the ten methods.

\section{Conclusions}

In this article, we introduce a more flexible extension of the Burr-Hatke distribution called inverse-power Burr-Hatke (IPBH) distribution that provides more accuracy and flexibility in fitting engineering and medicine data. The new model was generated based on the inverse-power transformation technique. The hazard rate function of the IPBH distribution exhibits an increasing shape, a decreasing shape, or an upside-down bathtub shape. The IPBH model can accommodate right-skewed shape, symmetrical shape, reversed J shape, and left-skewed shape densities. Some of its basic mathematical properties are derived. The two parameters of the IPBH distribution are estimated using ten classical and Bayesian estimation approaches. The behavior and performance of these estimators are explored using simulation results. We also determined the best estimation approach using partial and overall ranks for all estimators. As expected, the Bayesian method outperforms other classical methods under the different loss functions. The flexibility and practical importance of the IPBH distribution are explored empirically using two real-life datasets. It is shown that the IPBH distribution has a superior fit compared to the Burr-Hatke distribution and other competing models.

For some possible directions for future studies, the IPBH model can be modified with "polynomial variable transfer" to introduce new model with several free parameters which makes it attractive for analysis and approximation of specific data from different areas such as growth theory, test theory, biostatistics, and computer viruses propagation. Furthermore, different approximation problems related to the "saturation" in Hausdorff sense can be explored for the new model along with some numerical examples using CAS Mathematica to validate the results. More details about these directions can be explored in $[20,21]$.

Moreover, the T-X family may be applied to define the new inverse-power Burr-Hatke-G family of distributions. Several properties of this new family may be established, its special sub-models may be explored, and their applications in different applied fields may also be addressed.

\section{Data Availability}

This work is mainly a methodological development and has been applied on secondary data, but, if required, data will be provided.

\section{Conflicts of Interest}

The authors declare no conflicts of interest.

\section{Acknowledgments}

This study was funded by Taif University Researchers Supporting Project (no. TURSP-2020/279), Taif University, Taif, Saudi Arabia.

\section{References}

[1] Z. Ahmad, E. Mahmoudi, M. Alizadeh, R. Roozegar, and A. Afify, "The exponential T-X family of distributions: properties and an application to insurance data," Journal of Mathematics, vol. 2021, Article ID 3058170, 18 pages, 2021. 
[2] A. A. Al-Babtain, I. Elbatal, H. Al-Mofleh, A. M. Gemeay, A. Z. Afify, and A. M. Sarg, "The flexible Burr X-G family: properties, inference, and applications in engineering science," Symmetry, vol. 13, no. 3, p. 474, 2021.

[3] A. Z. Afify, G. M. Cordeiro, N. A. Ibrahim, F. Jamal, M. Elgarhy, and M. A. Nasir, "The Marshall-Olkin odd Burr III-G family: theory, estimation, and engineering applications," IEEE Access, vol. 9, pp. 4376-4387, 2021.

[4] A. Al-Babtain, R. A. K. Sherwani, A. Z. Afify et al., "The extended Burr-R class: properties, applications and modified test for censored data," AIMS Mathematics, vol. 6, no. 3, pp. 2912-2931, 2021.

[5] S. M. Zaidi, M. M. Sobhi, M. El-Morshedy, and A. Z. Afify, "A new generalized family of distributions: properties and applications," AIMS Mathematics, vol. 6, no. 1, pp. 456-476, 2021.

[6] M. E. Mead, "Generalized inverse gamma distribution and its application in reliability," Communications in StatisticsTheory and Methods, vol. 44, no. 7, pp. 1426-1435, 2015.

[7] S. H. Alkarni, "Extended inverse Lindley distribution: properties and application," SpringerPlus, vol. 4, no. 1, p. 690, 2015.

[8] V. K. Sharma, S. K. Singh, U. Singh, and V. Agiwal, "The inverse Lindley distribution: a stress-strength reliability model with application to head and neck cancer data," Journal of Industrial and Production Engineering, vol. 32, no. 3, pp. 162-173, 2015.

[9] H. S. Al-Kzzaz and M. M. E. Abd El-Monsef, "Inverse power Maxwell distribution: statistical properties, estimation and application," Journal of Applied Statistics, pp. 1-20, 2021.

[10] K. V. P. Barco, J. Mazucheli, and V. Janeiro, "The inverse power Lindley distribution," Communications in Statistics-Simulation and Computation, vol. 46, no. 8, pp. 63086323, 2017.

[11] A. S. Hassan and M. Abd-Allah, "On the inverse power Lomax distribution," Annals of Data Science, vol. 6, no. 2, pp. 259-278, 2019.

[12] A. Isaic-Maniu and V. G. h. Voda, "Generalized Burr-Hatke equation as generator of a homographic failure rate," Journal of Applied Quantitative Methods, vol. 3, no. 3, 2008.

[13] E. Chiodo, P. De Falco, L. Pio Di Noia, and F. Mottola, "Inverse log-logistic distribution for extreme wind speed modeling: genesis, identification and Bayes estimation," AIMS Energy, vol. 6, no. 6, pp. 926-948, 2018.

[14] P. L. Ramos, F. Louzada, T. K. O. Shimizu, and A. O. Luiz, "The inverse weighted Lindley distribution: properties, estimation and an application on a failure time data," Communications in Statistics-Theory and Methods, vol. 48, no. 10, pp. 2372-2389, 2019.

[15] S. A. Klugman, H. H. Panjer, and G. E. Willmot, Loss Models: From Data to Decisions, Vol. 715, John Wiley \& Sons, , Hoboken, NJ, USA, 2012.

[16] F. Louzada, P. L. Ramos, and D. Nascimento, "The inverse Nakagami-m distribution: a novel approach in reliability," IEEE Transactions on Reliability, vol. 67, no. 3, pp. 1030-1042, 2018.

[17] J. Galambos, The Asymptotic Theory of Extreme Order Statistics, R. E. Krieger Publishing, Malabar, FL, USA, 1987.

[18] M. R. Mahmoud and R. M. Mandouh, "On the transmuted fréchet distribution," Journal of Applied Sciences Research, vol. 9, no. 10, pp. 5553-5561, 2013.

[19] A. Gross and V. Clark, Survival Distributions: Reliability Applications in the Biomedical Sciences, John Wiley \& Sons, Hoboken, NJ, USA, 1975.
[20] N. Kyurkchiev, "A note on the Burr-Hatke-exponential model. some applications," Comptes Rendus de l'Academie Bulgare des Sciences: Sciences Mathématiques et Naturelles, vol. 74, pp. 488-495, 2021.

[21] N. Kyurkchiev and S. Markov, "On the Hausdorff distance between the heaviside step function and Verhulst logistic function," Journal of Mathematical Chemistry, vol. 54, no. 1, pp. 109-119, 2016. 